\title{
Positive Computing
}

Liebe Leserinnen, liebe Leser,

Wenn schon die Informationstechnologie ihren Anwender anstrengt und frustriert, muss man sich doch auch fragen, ob das notwendig ist, ob sie ihm nicht auch Spaß machen kann. Kann man nicht das Computing dahin bringen, dass es positive Gefühle auslöst? Eben diese Frage hat sich die Bewegung "Positive Computing"1 zum Forschungsthema gestellt. Das Ziel ist ein Paradigmenwechsel: Von der ersten nüchternen Phase zu einer zweiten lustvolleren Phase. Die Frustration an den Tücken des Computers soll überwunden und durch die zweite Phase, die des „Positive Computing", abgelöst werden. Der Anwender soll Spaß am Computing haben - Spaß als etwas, das man unmittelbar empfindet, bevor es von moralischen Rücksichten eingetrübt werden kann. Dabei mag man z.B. an die jungen Leute denken, die sich mit ihren Smartphones weltweit virtuelle Freunde beschaffen und im Verkehr mit ihnen und dem Internetangebot ihren Spaß haben.

Als ich kürzlich einen Beitrag2 zu „Positive Computing“ las, war mir - ehrlich gestanden - dieses Thema neu. Bei näherem Hinblicken stellte ich fest, dass der Begriff vielfältig verstanden wird und vieles umfasst. Alles, was am Computing eine positive Empfindung auslösen kann, lässt sich darunter subsumieren - etwa jede effektive Anti-Viren-Software, jede gelungene Mensch-Maschine-Schnittstelle, jede nützliche App. Wohl aber bleibt Spaß der Ansatz der Positive-Computing-Bewegung, der $\mathrm{Spa}$ an allem, was man in globalen Anwenderkreisen als angenehm, nützlich, anregend oder sonst wie positiv empfindet. Dabei geben die Gefühle des Anwenders den Ausschlag, und nicht das, was der Anbieter empfindet.

Bei der Lektüre des oben erwähnten Beitrags fiel mir der Datenschutz ein: Könnte Datenschutz als eine Art von Positive Computing gelten? Dafür spricht einiges. Der Datenschutz will menschlich Positives bezwecken. Er will das Computing dahin bringen, dass es dem Menschen die anonymen Gefahren des Computing deutlich macht und ihn wie ein Schutzengel um sie herum führt. Datenschutz erwirkt demnach den Fortschritt von einer Gesellschaft, die (von den Computing-Anbietern) transparent gehalten werden kann, zu einer Gesellschaft, die sich der damit verbundenen Gefahren bewusst ist; sich (vom Datenschutz) beschützt weiß oder glaubt. Schutz wirkt auf den Geschützten beruhigend, also gefühlt positiv. So verstanden, fällt Datenschutz unter Positive Computing.

Aber wo es auch sonst Gefühle gibt, da stellt sich auch Rücksicht auf Moral, Political Correctness, Volksweisheit etc. ein. Diese Instanzen hinterfragen den empfundenen Spaß und sorgen sich um die Folgen und das Ende. Solche Sorge kann den $\mathrm{Spa} ß$ an der Sache trüben. Gelegentlich überwiegt sie den Spaß und drückt inn beiseite. Sie gibt an, was als positiv zu empfinden ist und in der Gesellschaft Spaß machen darf. Das steht dann in seiner politischen Korrektheit übermächtig gegen den Spaß. Es kann inn verderben; das einerseits. Es kann aber auch gut und richtig sein, denn Spaß kann auch Schaden anrichten; die Nüchternheit kann sich als das Bekömmlichere herausstellen.

Ich glaube, liebe Leserinnen und Leser: In diese skeptische Ecke der Seele ordnet sich der Datenschutz ein. Er will zwar Positives, macht aber nicht Spaß; allein schon, weil er Gefahren bewusst und damit Angst macht. Das mag auch der Grund dafür sein, dass ich - zugegeben, mit unzureichender Mühe - in dem mir zum Thema „Positive Computing" Angelesenem nirgends den Datenschutz erwähnt fand.

Mit freundlichen Grüßen, Ihr

\section{Kail Rhavels}

1 Angeregt von Calvo, R. A., \& Peters, D. (2014). Positive computing: technology for wellbeing and human potential. Cambridge, Massachusetts: MIT Press.

2 Pawlowski, Eimler, Jansen, Stoffregen, Geisler, Koch, Müller, Handmann, Positive computing: A New Trend in Business and Information Systems Engineering? BISE 6/2015, Springer Gabler 